Article

\title{
Nanovectorized Microalgal Extracts to Fight Candida albicans and Cutibacterium acnes Biofilms: Impact of Dual-Species Conditions
}

\author{
Virginie Lemoine ${ }^{1}$, Clément Bernard ${ }^{1}$, Charlotte Leman-Loubière ${ }^{2}$, Barbara Clément-Larosière ${ }^{3}$, \\ Marion Girardot ${ }^{1}$, Leslie Boudesocque-Delaye ${ }^{2}$, Emilie Munnier ${ }^{4}\left(\mathbb{D}\right.$ and Christine Imbert ${ }^{1, *}$ \\ 1 Laboratoire Ecologie et Biologie des Interactions, Université de Poitiers, UMR CNRS 7267, \\ 86073 Poitiers, France; lemoinev60@gmail.com (V.L.); clement.bernard@univ-poitiers.fr (C.B.); \\ marion.girardot@univ-poitiers.fr (M.G.) \\ 2 Laboratoire SIMBA EA 7502, Faculté de Pharmacie, Université de Tours, 31 avenue Monge, \\ 37200 Tours, France; charlotte.leman-loubiere@clarins.com (C.L.-L.); \\ leslie.boudesocque@univ-tours.fr (L.B.-D.) \\ 3 Dénitral, Groupe COOPERL, 7 rue des Blossières Maroue BP 60328, 22403 Lamballe, France; \\ barbara.clement-larosiere@cooperl.com \\ 4 Laboratoire Nanomédicaments et Nanosondes EA 6295, Faculté de Pharmacie, Université de Tours, \\ 31 avenue Monge, 37200 Tours, France; emilie.munnier@univ-tours.fr \\ * Correspondence: christine.imbert@univ-poitiers.fr
}

Received: 30 April 2020; Accepted: 23 May 2020; Published: 26 May 2020

\begin{abstract}
Biofilm-related infections are a matter of concern especially because of the poor susceptibility of microorganisms to conventional antimicrobial agents. Innovative approaches are needed. The antibiofilm activity of extracts of cyanobacteria Arthrospira platensis, rich in free fatty acids, as well as of extract-loaded copper alginate-based nanocarriers, were studied on single- and dual-species biofilms of Candida albicans and Cutibacterium acnes. Their ability to inhibit the biofilm formation and to eradicate $24 \mathrm{~h}$ old biofilms was investigated. Concentrations of each species were evaluated using flow cytometry. Extracts prevented the growth of $C$. acnes single-species biofilms (inhibition $>75 \%$ at $0.2 \mathrm{mg} / \mathrm{mL}$ ) but failed to inhibit preformed biofilms. Nanovectorised extracts reduced the growth of single-species C. albicans biofilms (inhibition $>43 \%$ at $0.2 \mathrm{mg} / \mathrm{mL}$ ) while free extracts were weakly or not active. Nanovectorised extracts also inhibited preformed C. albicans biofilms by $55 \%$ to $77 \%$, whereas the corresponding free extracts were not active. In conclusion, even if the studied nanocarrier systems displayed promising activity, especially against $C$. albicans, their efficacy against dual-species biofilms was limited. This study highlighted that working in such polymicrobial conditions can give a more objective view of the relevance of antibiofilm strategies by taking into account interspecies interactions that can offer additional protection to microbes.
\end{abstract}

Keywords: antibiofilm; antimicrobial agent; bacteria; fungi; polymicrobial biofilm; microalga; free fatty acids; encapsulation

\section{Introduction}

Biofilms are involved in numerous diseases, both superficial and systemic, for instance those affecting the oral cavity, skin or related to an implanted medical device. They can be single species, but most often they are polymicrobial and contain both fungi and bacteria. For example, dermal wounds are colonized by aerobic and anaerobic bacterial and fungal species, most of them belonging to resident microbiota of the surrounding skin, oral cavity and gut, or from the external environment [1]. It has been shown that $60 \%$ of chronic wounds exhibit a biofilm which is a major factor in delayed 
wound healing [2-5]. Also, it is considered that Candida spp. are among the primary causes of delayed healing and infection in both acute and chronic wounds, especially those of a surgical nature $[6,7]$. Literature data suggest that Candida spp. rarely colonize human skin but can cause infection especially in specific conditions such as immune deficiency, diabetes or after antibiotic use [8,9].

Gram-positive bacteria Cutibacterium acnes (formerly Propionibacterium acnes) [10] are a main colonizer and inhabitant of the skin [11,12] and a good biofilm former, producing both single species and polymicrobial biofilms [13-16]. Its involvement in chronic skin disease such as acne vulgaris is very well-known and this species is also occasionally involved in non-skin-related infections such as prosthetic joint infections, some of them being related to the formation of a biofilm $[17,18]$. As for many other microbial species, sessile C. acnes cells as well as Candida albicans cells have been shown to be more tolerant to conventional antibiotics than their planktonic counterparts [19-21].

Our team recently showed that $C$. acnes and C. albicans can form dual-species biofilms, with C. acnes adhering to both hyphal and yeast forms of $C$. albicans [15]. The presence of metabolically active C. albicans cells enhanced the early growth of $C$. acnes under aerobic conditions, while no influence was observed in anaerobic conditions. We also recently demonstrated that the co-presence of these species in biofilms influenced their sensitivity to micafungin, a major conventional antifungal agent. Actually, C. acnes was shown to protect $C$. albicans cells from the effect of micafungin in dual-species biofilms [22]. Along the same lines, Montelongo-Jauregui et al. showed that the resistance of C. albicans to amphotericin B and caspofungin, as well as the resistance of Streptococcus gordonii to clindamycin were increased due to a dual-species biofilm produced by C. albicans and with Streptococcus gordonii, compared to single-species conditions [23]. Therefore, a double issue should be thus observed-biofilm lifestyle causes itself a decreased susceptibility to antimicrobial agents and the polymicrobial nature of the biofilm can make this lack of susceptibility even worse.

Biofilms are infectious reservoirs and the most effective way to prevent biofilm-related infections requires the eradication of these complex microbial structures, that is their detachment, their disorganization and the killing of all released microbial cells, with these three events needing to be concomitant. Unfortunately, the available antimicrobial conventional molecules fail to reach this challenging goal.

Free fatty acids (FFAs) are physiological antimicrobial agents occurring on skin, exhibiting a wide antimicrobial spectrum (antibacterial, antifungal, antibiofilm ... ) [24,25]. Microalgae have been well-described as abundant sources of lipids and especially FFAs [26,27]. Those FFAs, especially polyunsaturated (PUFAs), may also represent a potential source of topical drugs against polymicrobial biofilms. Indeed, a previous screening of 29 FFAs based on topical antibacterial activity highlighted that PUFAs were among the most active [25]. Arthrospira platensis (formerly Spirulina platensis) appeared as a good model among all microalgae as it was the most studied microalgae with a well-known FFA profile.

Due to their lipid nature, FFAs are not able to penetrate the biofilm made of highly hydrophilic exopolysaccharide. Recently, nanosized systems showed their ability to vectorize active molecules in biofilms. Core-shell nanosystems, with a hydrophilic shell and a lipophilic core, seem to be very appropriate vectors for low-polarity active molecules [28,29]. Alginate-based nanocarriers, nanosystems made of a triglyceride core and an alginate gel shell, were shown to be efficient to vectorize FFAs in C. albicans biofilm [30]. The reproducibility of the preparation, the stability of the systems and their FDA-approved ingredients constitute key advantages for their use in dermatology. Moreover, they were shown to be stable in dermatological preparations [31].

We previously developed a proof of concept of the potential of A. platensis extracts and alginate-based nanocarriers combination as a possible strategy to fight $C$. albicans single-species biofilms. The antibiofilm strategies are all the more innovative and promising in that they are able to act on taxonomically distant and diverse microbial species, because of the polymicrobial nature of most biofilms developing in humans. 
Thus, this current study aimed to obtain lipid extracts from Cyanobacteria A. platensis, to develop extract-loaded copper alginate-based nanocarriers able to carry a lipid extract and to evaluate the antibiofilm activity of these lipid extracts nanovectorized or free, against both single-species fungal and bacterial biofilms and interkingdom dual-species biofilms.

\section{Results}

\subsection{A. platensis Extraction}

A. platensis biomass was extracted using two sustainable solvents-EtOAc and DMC. Resulting extracts were enriched in lipids (Table 1), with a closely related FFA profile. Both extracts contained mainly $\omega 6$ PUFA, i.e., linoleic and $\gamma$-linolenic acid (more than $60 \%$ of total FFAs) (Table 2). Also, lipophilic dyes (chlorophyll and carotenoids) were co-extracted (Table 2), but their content remained low, highlighting again the good selectivity of these solvents towards lipids.

Table 1. A. platensis extracts composition (total lipids, chlorophylls, carotenoids).

\begin{tabular}{ccc}
\hline Extract & EtOAc & DMC \\
\hline Total lipids (mg of equiv. castor oil/g of extract) & $1115.1 \pm 87.2$ & $980.3 \pm 67.9$ \\
\hline Chlorophylls (mg/g of extract) & $82.0 \pm 8.1$ & $52.6 \pm 2.5$ \\
\hline Carotenoids (mg/g of extracts) & $53.5 \pm 8.1$ & $61.0 \pm 2.6$ \\
\hline
\end{tabular}

Table 2. Free fatty acid (FFA) ratios in A. platensis extracts, in relative percentage of total FFA.

\begin{tabular}{ccc}
\hline & EtOAc & DMC \\
\hline Saturated & & \\
\hline Myristic acid & nd & nd \\
Palmitic acid & $27.0 \%$ & $21.9 \%$ \\
Stearic acid & $5.1 \%$ & $5.7 \%$ \\
\hline MUFA & & \\
\hline Myristoleic acid & nd & nd \\
Palmitoleic acid & nd & nd \\
Oleic acid & $4.8 \%$ & $4.8 \%$ \\
\hline PUFA & & \\
\hline Linoleic acid & $41.1 \%$ & $42.3 \%$ \\
$\gamma$-Linolenic acid & $22.0 \%$ & $25.2 \%$ \\
\hline
\end{tabular}

nd $=$ nondetected $n=1$.

\subsection{A. platensis Extracts Vectorization}

Extract-loaded ANCs were prepared with EtOAc extract and DMC extract. Physicochemical characteristics are shown in Table 3.

Table 3. Physicochemical characteristics of extract-loaded alginate-based nanocarriers.

\begin{tabular}{cccc}
\hline & $\begin{array}{c}\text { Hydrodynamic Diameter } \\
(\mathbf{n m}) \mathbf{( M e a n} \pm \mathbf{S D}, \boldsymbol{n = 3})\end{array}$ & $\begin{array}{c}\text { Polydispersity Index } \\
(\text { Mean } \pm \mathbf{S D}, \boldsymbol{n}=\mathbf{3})\end{array}$ & $\begin{array}{c}\text { Zeta Potential }(\mathbf{m V}) \\
\text { (Mean } \pm \mathbf{S D}, \boldsymbol{n}=\mathbf{3})\end{array}$ \\
\hline Empty ANC & $249 \pm 9$ & $0.129 \pm 0.044$ & $-24.5 \pm 0.7$ \\
EtOAc extract-loaded ANC & $236 \pm 2$ & $0.147 \pm 0.018$ & $-24.2 \pm 0.2$ \\
DMC-extract loaded ANC & $250 \pm 1$ & $0.139 \pm 0.015$ & $-24.1 \pm 0.9$ \\
\hline
\end{tabular}

Extract-loaded ANCs show similar size and surface potential as empty ANCs with a pure Labrafac $^{\circledR \circledR}$ WL 1349 core. The polydispersity index lower than 0.2 shows a monodispersity of 
the suspensions, guaranteeing the reproducibility of the dosage. The negative surface charge of the nanocarriers participates to the colloidal stability of the nanocarriers and should not limit their interaction with the biofilms. Indeed, even if biofilms are generally considered negatively charged and could thus bind more easily to cationic nanoparticles [32], several negatively charged systems displayed antibiofilm efficacy $[28,33]$. The native ANC suspension shows a concentration in A. platensis extract of $\sim 1 \mathrm{mg} / \mathrm{mL}$.

\subsection{Ability of A. platensis Extracts to Prevent Biofilm Formation}

In single-species conditions, EtOAc extract used at $0.2 \mathrm{mg} / \mathrm{mL}$ displayed a significant $(p=0.0001)$ but very limited antibiofilm formation effect against $C$. albicans (24.4\% inhibition) (Figure $1 \mathrm{~A})$. This extract used at $0.1 \mathrm{mg} / \mathrm{mL}$ was not active against C. albicans, and DMC extracts (at both 0.1 and $0.2 \mathrm{mg} / \mathrm{mL}$ ) as well. Both EtOAc and DMC extracts significantly reduced the growth of $C$. acnes biofilms, regardless of the tested concentrations-inhibition ranged between $66.0 \%$ and $78.4 \%$ (EtOAc extract, $p \leq 0.003$ ) and between $67.6 \%$ and $86.2 \%$ (DMC extract, $p \leq 0.0008$ ) (Figure 1C). However, no real conclusion can be made in the case of EtOAc $(0.1$ and $0.2 \mathrm{mg} / \mathrm{mL})$ and DMC $(0.1 \mathrm{mg} / \mathrm{mL})$ as the error bars are very high.

A

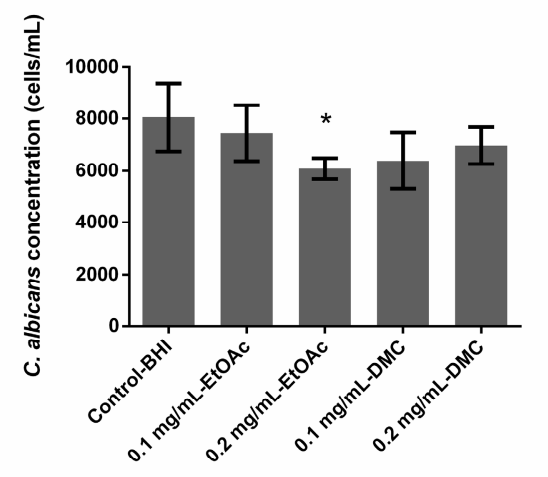

C

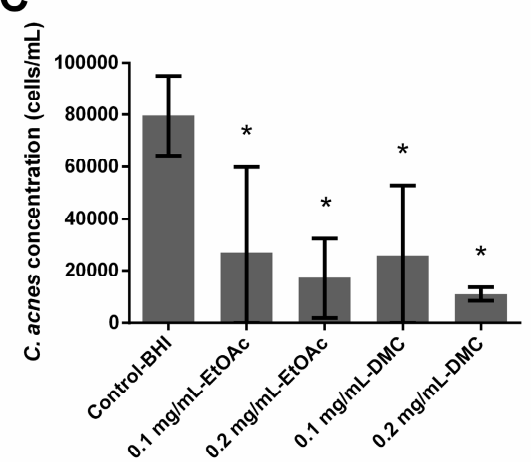

B
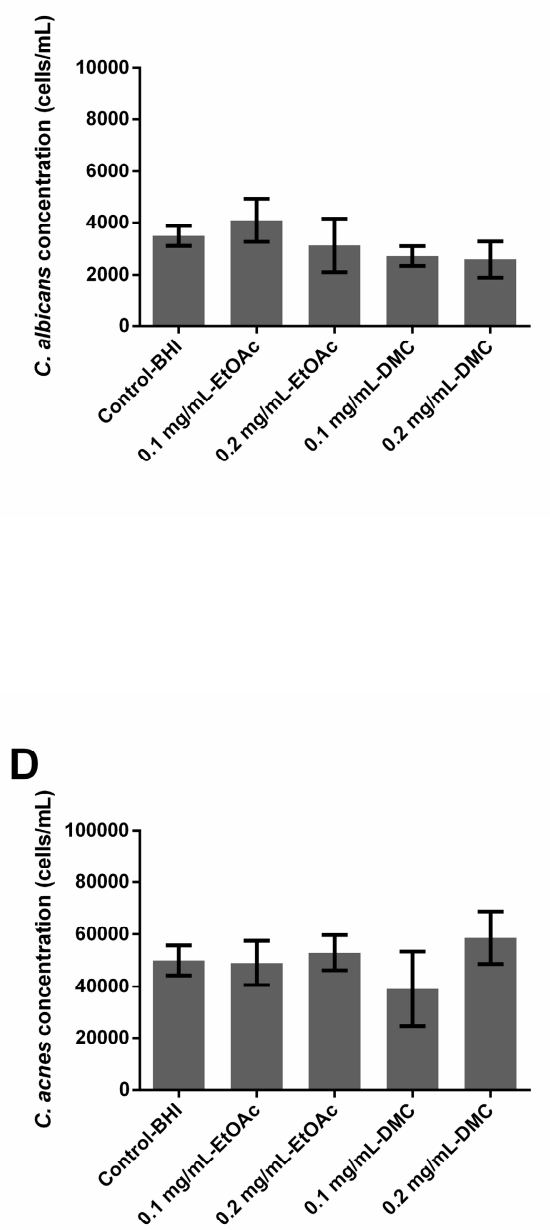

Figure 1. Ability of Arthrospira fusiformis extracts to prevent biofilm formation. Single-species biofilms (C. albicans) (A); C. albicans concentration obtained in dual-species biofilms (C. albicans + C. acnes) $(\mathbf{B})$; single-species biofilms (C. acnes) (C); C. acnes concentration obtained in dual-species biofilms (C. albicans + C. acnes) $(\mathbf{D})$. Results are expressed as mean \pm SD. ${ }^{*} p<0.005$ : test condition vs. BHI-control (biofilms treated with BHI only). 
In dual-species conditions, neither EtOAc nor DMC extract solutions were able to reduce the biofilm formation of $C$. albicans and no reduction was observed in the fungal and bacterial populations.

\subsection{Ability of A. platensis Extracts to Eradicate Preformed Biofilms}

None of the extracts, whatever the tested concentration, had any effect on C. albicans or C. acnes preformed single-species or dual-species biofilms. No reduction was observed in the fungal and bacterial populations after a $24 \mathrm{~h}$ treatment (Figure 2).

A

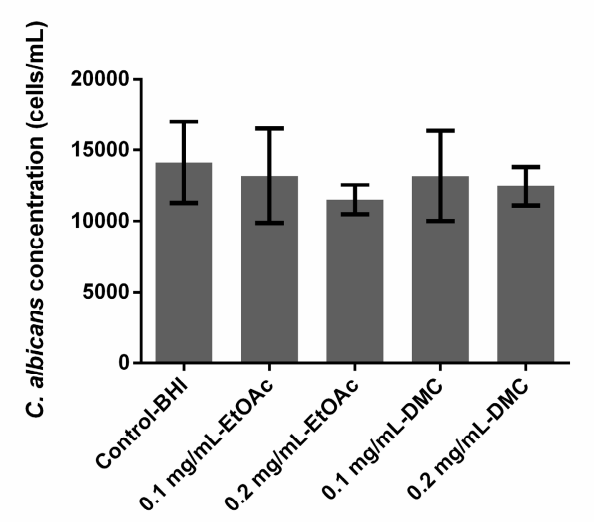

C

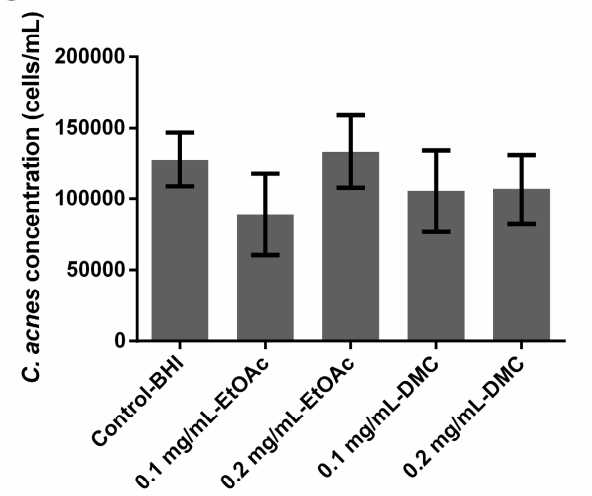

B

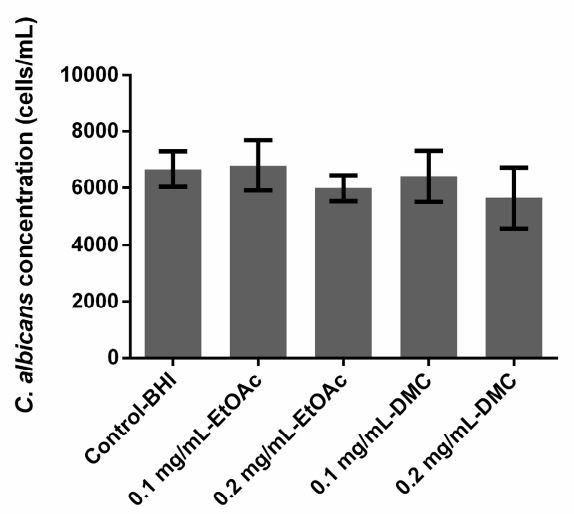

D

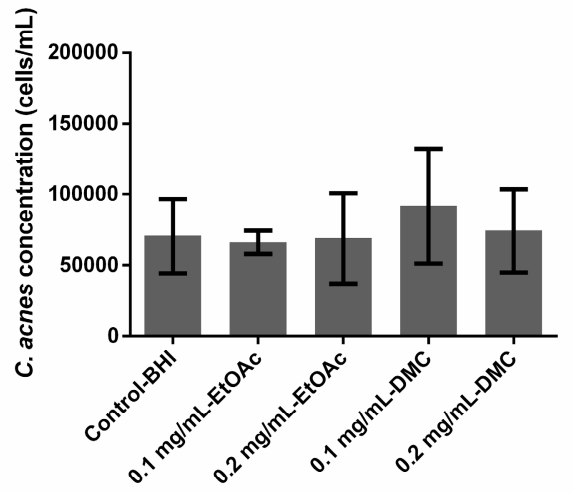

Figure 2. Ability of Arthrospira fusiformis extracts to eradicate preformed biofilm. Single-species biofilms (C. albicans) (A); C. albicans concentration obtained in dual-species biofilms (C. albicans + C. acnes) (B); single-species biofilms (C. acnes) (C); C. acnes concentration obtained in dual-species biofilms (C. albicans + C. acnes) (D). Results are expressed as mean \pm SD. ${ }^{*} p<0.005$ : test condition vs. BHI-control (biofilms treated with BHI only).

\subsection{Ability of A. platensis Extracts Encapsulated in Alginate-Based Nanocarriers to Prevent Biofilm Formation}

In single-species conditions, empty nanocarriers inhibited the growth of $C$. albicans biofilms by $51.55 \%\left(0.1 \_\right.$emptyNC, $\left.p=0.001\right)$ or $54.14 \%\left(0.2 \_\right.$emptyNC, $\left.p=0.0002\right)$, while they had no effect on C. acnes biofilms (Figure 3A,C). Nanocarriers loaded with extract solutions at $0.2 \mathrm{mg} / \mathrm{mL}$ $\left(0.2 \mathrm{mg} / \mathrm{mL} \_E E N C\right)$ inhibited the growth of C. albicans biofilms by $51.35 \%$ (EtOAc, $\left.p=0.0031\right)$ or $43.77 \%$ (DMC, $p=0.0021)$ while those loaded with extract solutions at $0.1 \mathrm{mg} / \mathrm{mL}(0.1 \mathrm{mg} / \mathrm{mL}$ EENC) had no significant influence. Regarding the growth of $C$. acnes biofilms, only nanocarriers loaded with EtOAc 
extract at $0.2 \mathrm{mg} / \mathrm{mL}$ and DMC extract at $0.1 \mathrm{mg} / \mathrm{mL}$ demonstrated a weak inhibitory activity of $22.48 \%$ ( $p=0.0016)$ and $32.74 \%(p=0.0004)$, respectively (Figure 3C).

A
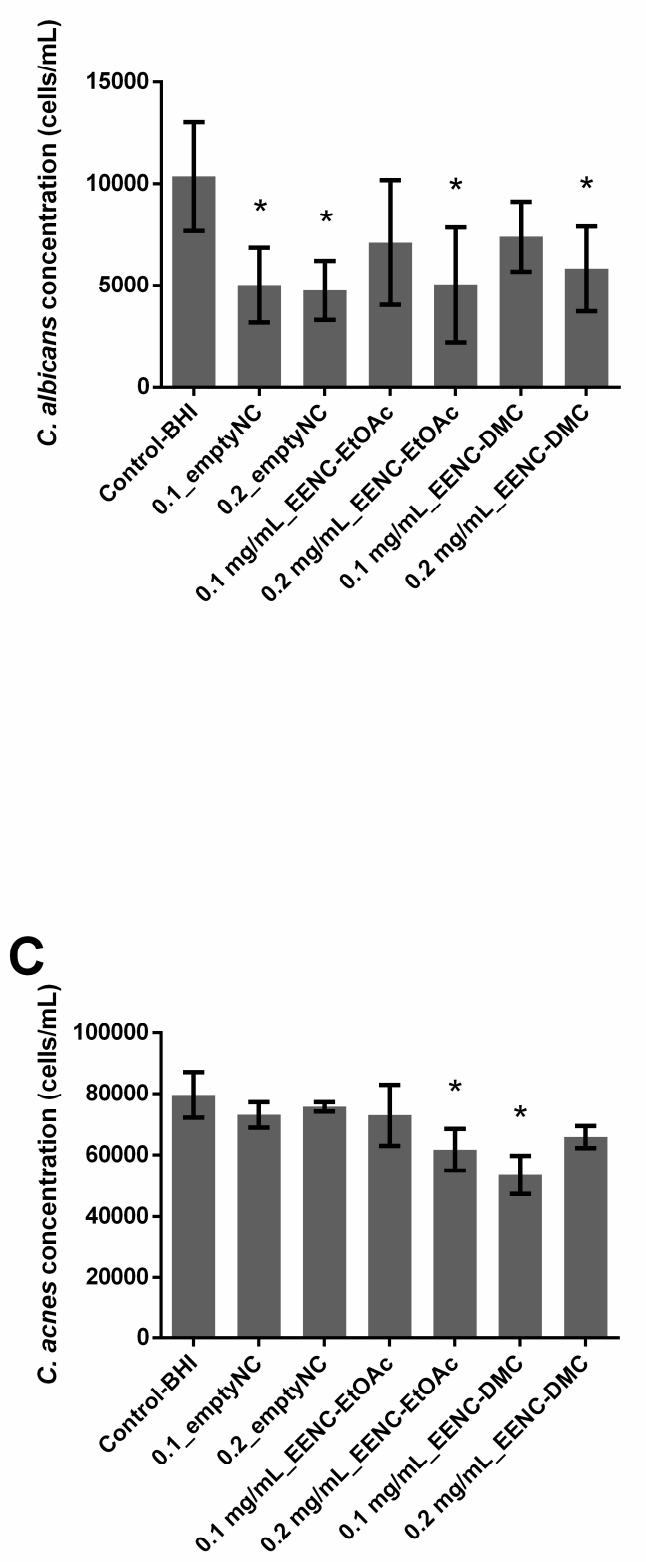

B
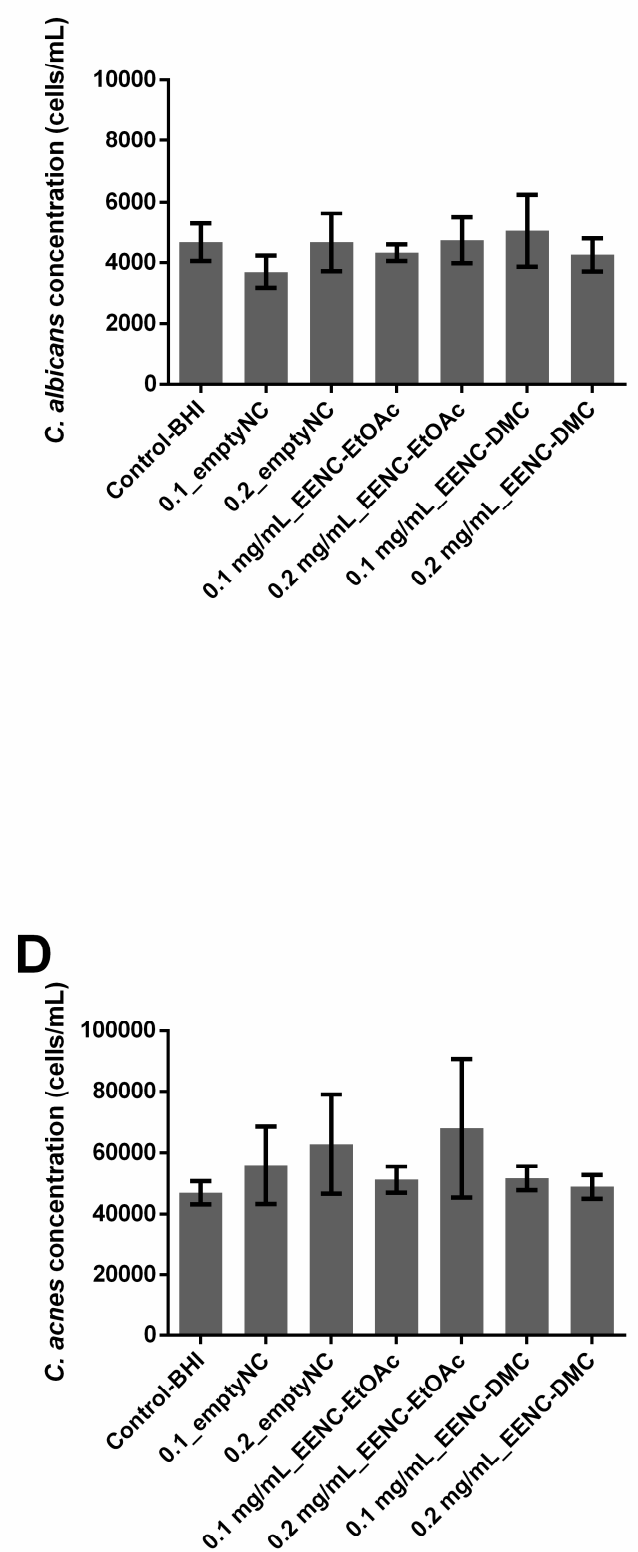

Figure 3. Ability of Arthrospira fusiformis extracts encapsulated in alginate nanocarriers to prevent biofilm formation. Single-species biofilms (C. albicans) (A); C. albicans concentration obtained in dual-species biofilms (C. albicans + C. acnes) (B); single-species biofilms (C. acnes) (C); C. acnes concentration obtained in dual species biofilms (C. albicans + C. acnes) (D). Results are expressed as mean \pm SD. ${ }^{*} p<0.005$ : test condition vs. BHI-control (biofilms treated with BHI only).

In dual-species conditions, nanocarriers loaded with extract solutions did not limit the growth of either C. albicans or C. acnes in biofilms. Only empty nanocarriers (0.1_emptyNC, $p=0.0046)$ displayed a weak activity but were not significant $(p>0.005)$ against $C$. albicans growth $(21.0 \%)$ and no reduction was observed on C. acnes population (Figure 3B,D). 
2.6. Ability of A. platensis Extracts Encapsulated in Alginate-Based Nanocarriers to Eradicate Preformed Biofilms

In single-species conditions, empty nanocarriers inhibited preformed biofilms of C. albicans by $58.7 \%\left(0.1 \_\right.$emptyNC, $\left.p<0.0001\right)$ or $76.69 \%(0.2$ emptyNC, $p<0.0001)$, whereas they had no effect on $C$. acnes biofilms (Figure 4A,B). Whatever the conditions, all nanocarriers loaded with extract solutions inhibited preformed single species C. albicans biofilms $(p<0.0001)$ by at least $55 \%$; nanocarriers loaded with EtOAc extracts inhibited biofilms by $76.9 \%(0.1 \mathrm{mg} / \mathrm{mL}$ EENC-EtOAc) and $62.35 \%$ (0.2 mg/mL_EENC-EtOAct) whereas those loaded with DMC extracts induced a 55.69\% $\left(0.1 \mathrm{mg} / \mathrm{mL} \_\right.$EENC-DMC) and a $77.32 \%(0.2 \mathrm{mg} / \mathrm{mL}$ EENC-DMC) inhibition. On the contrary, whatever the conditions, both empty and loaded nanocarriers failed to significantly reduce an already formed single-species biofilm of C. acnes ( $p>0.005)$ (Figure $4 C)$.

A
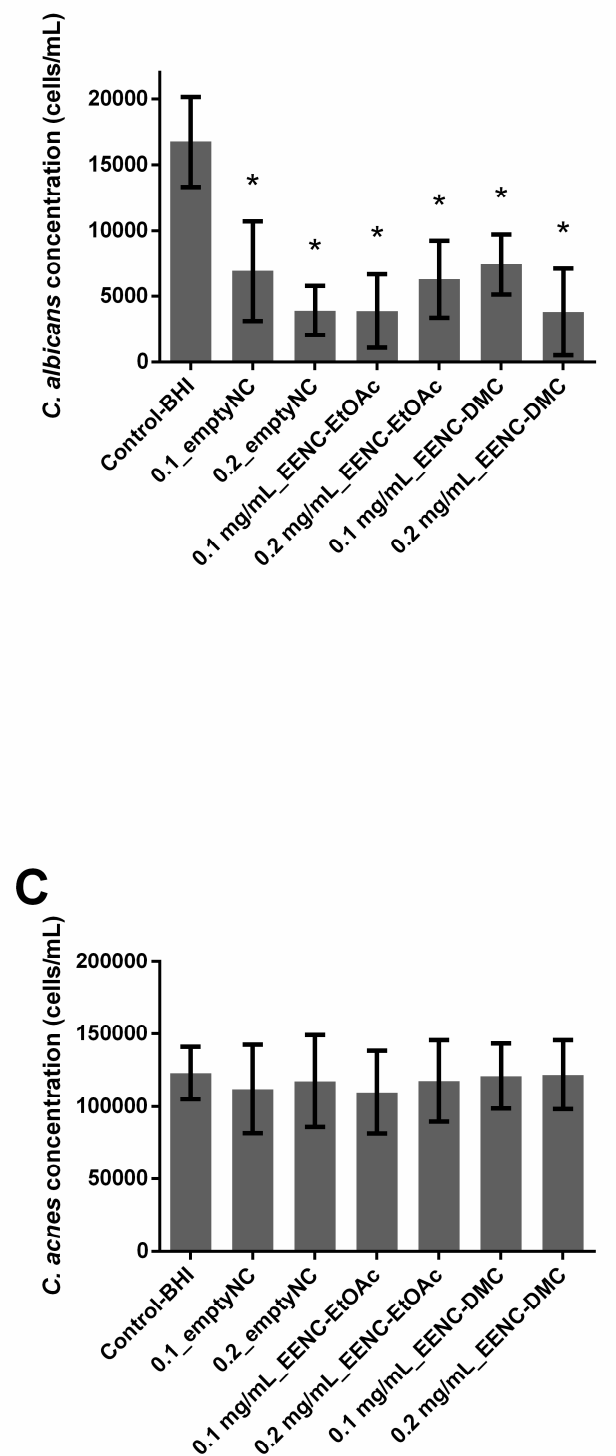

B
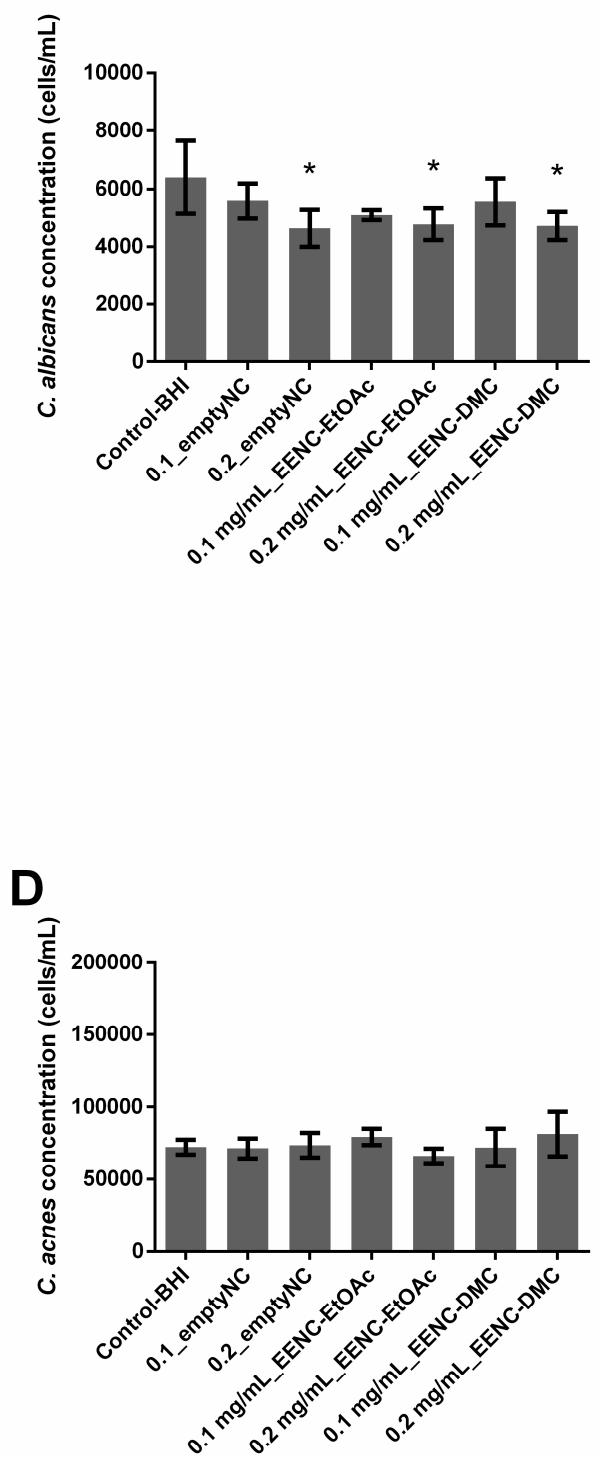

Figure 4. Ability of Arthrospira fusiformis extracts encapsulated in alginate nanocarriers to eradicate preformed biofilm. Single-species biofilms (C. albicans) (A); C. albicans concentration obtained in dual-species biofilms (C. albicans + C. acnes) (B); single-species biofilms (C. acnes) (C); C. acnes concentration obtained in dual-species biofilms (C. albicans + C. acnes) (D). Results are expressed as mean \pm SD. ${ }^{*} p<0.005$ : test condition vs BHI-control (biofilms treated with BHI only). 
In dual-species conditions, empty nanocarriers as well as those loaded with extract solutions induced inhibition always less than $28 \%$ of already formed dual-species biofilms, whatever the target population (C. albicans or C. acnes) (Figure 4B,D). Significant $p$-values demonstrating an inhibition of the $C$. albicans population were only observed in the case of 0.2_emptyNC (inhibition: 27.5\%), $0.2 \mathrm{mg} / \mathrm{mL} \_E E N C-E t O A c$ (inhibition: $25.3 \%$ ) and $0.2 \mathrm{mg} / \mathrm{mL} \_E E N C-D M C$ (inhibition: $26.3 \%$ ) (Figure 4B).

\section{Discussion}

The results are in accordance with those previously obtained when studying the activity of EtOAc extract at $0.2 \mathrm{mg} / \mathrm{mL}$ on C. albicans biofilms [30]. The ability of EtOAct extract at $0.2 \mathrm{mg} / \mathrm{mL}$ to inhibit C. albicans biofilms growth evidenced by the significant decrease in the number of cells forming treated biofilms (FCM approach) (Figure 1A) agrees with previous results showing that this extract was able to reduce the metabolic activity of $C$. albicans forming treated biofilms (XTT method). However, EtOAc extract at $0.1 \mathrm{mg} / \mathrm{mL}$ and DMC extract at $0.1 \mathrm{or} 0.2 \mathrm{mg} / \mathrm{mL}$ did not manage to decrease yeast concentration in biofilms, although they previously showed antimetabolic activity. The XTT method is a classical method used to quantify fungal biofilms [34-36]. However, this method does not allow a differentiation between bacterial and fungal populations in dual-species biofilms. That is why the FCM approach used for the current study was recently developed [22,37]. A comparative study previously suggested that results provided by colony-forming unit (CFU) counts, XTT reduction or FCM counts were generally comparable and occasional differences could be explained by the specificity and targets of each method [37]. For example, metabolic activity can be reduced without any change in the cell number explaining some divergence in XTT versus CFU or FCM count results. Slight differences between previous and present results could also be at least partially explained by the fact that two different $A$. platensis biomasses were used in these studies, leading to different compositions of extracts. Growth conditions impact the FFA profile as large amounts of $\omega 6$-MUFAs and PUFAs were highlighted here, with decreased rates of saturated FFAs, the latter being known to exhibit higher antifungal activity.

By comparing results obtained from growing biofilms (prophylactic activity) and preformed ones (curative activity), we observed that EtOAc extract at $0.2 \mathrm{mg} / \mathrm{mL}$ loses its activity once the biofilm is formed (Figures 1A and 2A). Similarly, although all tested extracts significantly limited the growth of single species $C$. acnes biofilms, they were not active anymore once the biofilm was preformed (Figures $1 \mathrm{C}$ and $2 \mathrm{C}$ ). The extracts, whether free or nanovectorized, were not active against dual-species biofilms, growing or already formed as well (Figure 1C,D and Figure 2B,D). Moreover, since single-species C. albicans biofilms were prepared aerobically and those involving C. acnes anaerobically, a role of the presence of oxygen could not be excluded to explain the different levels of antibiofilm activity that have been observed. In fact, the mechanism of action of the FFA is not completely elucidated. Some studies suggested that their antimicrobial activity would be partly explained by the formation of PUFA peroxidation products [24], which would be favored in an aerobic environment. These oxidized metabolites would act according to a mechanism different from that of native FFAs [24], explaining the residual activity observed on $C$. acnes. As we could expect, these results suggest that preventing the formation of a biofilm is easier than eradicating this biofilm once it is formed.

Different teams demonstrated that biofilms made of more than one species presented reduced susceptibility to antimicrobial treatment compared to single-species biofilms [38,39]. In addition to studying the activity of the extracts and nanocarriers loaded or not by extracts on single-species biofilms, our work assessed the impact of the dual-species nature of the biofilms. Indeed, our results showed that nanocarriers loaded or otherwise with A. platensis EtOAc extracts or loaded or not with A. platensis DMC extracts as well significantly reduced both already formed and formation of $C$. albicans single-species biofilms, but displayed no or poor activity against $C$. albicans in dual-species biofilms (Figure 3; Figure $4 \mathrm{~A}, \mathrm{~B})$. These results thus suggest that $C$. albicans growing with $C$. acnes in dual-species biofilms is more difficult to inhibit than in single-species ones, which agrees with previous studies on the efficacy of micafungin against $C$. albicans in these two conditions [22]. More generally, results published in recent 
years suggest that bacteria and fungi from dual-species biofilms such as C. albicans-Staphylococcus spp. or C. albicans-Streptococcus spp. often exhibit reduced susceptibilities towards antibiotic or antifungal agents, which is at least partially caused by their synergistic interaction [23,38,40-43]. This study confirmed the activity of the empty nanocarriers against $C$. albicans biofilms which was already observed by Boutin et al. in 2019 [30], suggesting that copper ions could efficiently reach C. albicans cells through this single-species biofilm. Cheong et al., 2020 recently confirmed that copper displayed a high antifungal activity against $C$. albicans [44]. Unfortunately, we observed that empty nanocarriers lose their activity at least partially against $C$. albicans as soon as $C$. acnes is present in biofilms, whatever the age of the studied biofilm. Punniyakotti et al. 2020, recently reported the antibiofilm activity of copper nanoparticles studying Pseudomonas and Staphylococcus species [45]. They hypothesized that $\mathrm{Cu}^{2+}$ ions liberated from the nanoparticles would be engrossed by the bacterial cell surface and cause cell damage, affecting biofilm development. These authors suggested that the surface binding capability of copper ions would play a key role in the biofilm inhibition. Although we can hypothesize a similar mechanism to explain the activity against fungi, there is no clear explanation as to why empty nanocarriers failed to inhibit biofilm in the presence of $C$. acnes. Nanocarriers loaded with $A$. platensis extracts failed to significantly prevent the formation of C. acnes biofilms whereas A. platensis extracts without nanocarriers did it in the range of $66.0 \%$ to $86.2 \%$. As empty nanocarriers display no activity either, we can hypothesize that nanocarrier loading would counteract the action of extracts against these bacteria (Figure 1; Figure 3C). Conversely, the encapsulation of $A$. platensis extracts induced up to $51.35 \%$ of inhibition against the formation of $C$. albicans single-species biofilms (Figures $1 \mathrm{~A}$ and 3A). As the empty nanocarriers inhibited C. albicans single-species biofilm formation and eradicated biofilms, the activity cannot be totally attributed to the extracts. Unfortunately, this encapsulation did not allow the growth inhibition of dual-species biofilms, whatever the studied species (Figure 1; Figure 3B,D).

Finally, A. platensis extracts alone or encapsulated in nanosystems displayed an absence of activity against $C$. acnes preformed biofilms (Figures 2C and 4C) whereas the encapsulation of $A$. platensis extracts gave a promising activity against $C$. albicans preformed single-species biofilms, inducing inhibition up to $77.32 \%$ (Figures $2 \mathrm{~A}$ and $4 \mathrm{~A}$ ). Whatever the microorganism studied, the encapsulation does not lead to the obtention of an efficient and significant inhibition of preformed dual-species biofilms (Figure 2B,D and Figure 4B,D)

Very few authors compared the effect of nanosystems vectorizing antimicrobial agents on monoor multispecies biofilms [46,47], and even less on biofilms mixing Gram-positive bacteria and fungi. It is now established that the efficacy of nanosystems on biofilms is linked to their capacity for deeply penetrating the matrix [32]. However, the penetration of nanoparticles into biofilms is highly dependent on the surface characteristics of the nanoparticles $[46,48]$. Our results suggest that ANCs can diffuse through the extracellular polymeric substance (EPS) of C. albicans biofilm, but are not able to diffuse in the EPS of $C$. acnes and in that of polymicrobial biofilm matrix as well. Anjum et al. showed that PLGA nanoparticules loaded with xylitol successfully penetrated into the EPS matrix of single-species biofilms of S. aureus or Pseudomonas aeruginosa, and also of dual-species biofilms [46]. In the study of Anjum et al., penetration was made easier by adding a ligand onto the nanoparticle surface targeting the biofilm matrix. Tan et al. measured the antibiofilm activity of nanoparticles including enzymes targeting the matrix of biofilms composed of S. aureus and C. albicans [47]. The particles were able to disrupt in a similar manner single-species or dual-species biofilms, but it was observed that adhesion of bacteria to Candida hyphae made their surface less accessible to antimicrobial molecules. This obstacle was already described for free antimicrobial molecules in dual-species biofilms of $S$. aureus and Fusarium falciforme [49]. This interaction between the microbial species was also observed for C. acnes et C. albicans [22] and could participate in the loss of activity of ANCs on C. albicans in the dual-species biofilm.

In conclusion, our results highlight the interest of $A$. platensis extracts in preventing the formation of $C$. acnes single-species biofilms. They also suggest that even if the nanocarrier developed by our 
team offers interesting features, especially in the case of C. albicans, its activity against dual-species biofilms is much more limited at the concentrations tested. Even if in vitro models represent simplified models, far from real clinical conditions, developing polymicrobial conditions gives a more realistic representation of clinical biofilms that develop in the human body. This study clearly demonstrated the impact of polymicrobial conditions on the antibiofilm efficacy of nanovectorized antimicrobial systems and highlighted the importance of working in such polymicrobial conditions to have a more objective view of the tested molecules or systems.

\section{Materials and Methods}

\subsection{Chemicals}

Ethyl acetate (EtOAc), methanol $(\mathrm{MeOH})$, toluene, hexane, formic acid, diethylether, glacial acetic acid, petroleum ether, sulfuric acid $96 \%\left(\mathrm{H}_{2} \mathrm{SO}_{4}\right)$ and dimethylsulfoxide (DMSO) were purchased from Carlo Erba (Val de Reuil, France). Dimethyl carbonate (DMC), sodium alginate, $( \pm$ )- $\alpha$-tocophérol 96\% (vitamin E), oleic acid, linoleic acid, palmitic acid, myristic acid, stearic acid, palmitoleic acid, $\gamma$-linolenic acid, 2,3-bis-(2-methoxy-4-nitro-5-sulfophenyl)-2H-tetrazolium-5-carboxanilide (XTT), menadione and glucose were purchased from Sigma Aldrich (Saint-Quentin Fallavier, France). Phosphoric acid 85\% was purchased from Merck pro analysis (Darmstad, Germany). Labrafac ${ }^{\circledR}{ }^{\circledR}$ WL 1349 was purchased from Gattefossé (Saint-Priest, France). Montane $80^{\circledR}$ and Montanox $80^{\circledR}$ were purchased from Seppic (Castres, France). Copper nitrate $\mathrm{Cu}\left(\mathrm{NO}_{3}\right)_{2}$ was purchased from Fisher Scientific SAS (Illkirch, France). Vanillin, acetic acid trihydrate 99+\% were purchased from Acros Organics (Geel, Belgium). Water was purified using a Milli-Q system (Millipore Corporation, Bedford, MA, USA).

\subsection{Biomass}

Arthrospira platensis was cultivated and harvested by DENITRAL SA (Lamballe, France) and kindly provided by Dr Barbara Clément-Larosière.

\subsection{Extraction Protocol and Extracts Analyses}

A total of $1 \mathrm{~g}$ of biomass was extracted with DMC or EtOAc according to the protocol described by Boutin et al. [30]. The calibration curve was built up using castor oil and results were expressed as mg of equivalent of castor oil in the extract.

Pigments and total lipid rates were obtained using protocol described in Boutin et al. (2019) [30]. FFA profiles were obtained using the LC-ESI-MS protocol adapted from Samburova et al. (2013) [50]. Briefly, LC-ESI-MS analyses were performed on an Acquity H-Class with an SQD detector (Waters, Saint Quentin en Yvelines, France). The system was fitted with a BEH C18 $(50 \times 2.1 \mathrm{~mm} ; 1.7 \mu \mathrm{m}$ particle size). The column oven was set at $40{ }^{\circ} \mathrm{C}$. Mobile phases were A Water $0.1 \% \mathrm{NH}_{3}$ aq; B acetonitrile $0.1 \%$ $\mathrm{NH}_{3}$ aq. Flow rate was $0.25 \mathrm{~mL} / \mathrm{min}$ and the gradient was set as follows-initial solvent $\mathrm{B}$ content was $10 \%$, raised to $40 \%$ in $2 \mathrm{~min}, 90 \%$ in $23 \mathrm{~min}$ and $100 \%$ in $1 \mathrm{~min}$ and maintained for $9 \mathrm{~min}$. ESI in negative mode was performed with cone voltage set at $50 \mathrm{~V}$ and capillary voltage at $2.8 \mathrm{kV}$.

\subsection{Alginate-Based Nanocarriers Preparation and Characterization}

Alginate-based nanocarriers (ANCs) were prepared using ultrasound oil-in-water emulsification followed by surface gelation with cupric ions inspired by Nguyen et al. [31] and adapted by Boutin et al. [30]. Briefly, an A. platensis lipid extract solution in Labrafac ${ }^{\circledR}$ WL $1349(6 \mathrm{mg} / \mathrm{mL}) \mathrm{was}$ emulsified with a sodium alginate solution in presence of nonionic surfactant, using an ultrasonic probe (Vibra-cell ultrasonic processor, Sonics, Newtown, CT, USA, $20 \mathrm{kHz}$ ). The resulting nanoemulsion was mixed under ultrasounds stirring with a solution of copper ions, which complex alginates to form an insoluble copper-alginate gel at the surface of the nanodroplets.

The hydrodynamic diameter and polydispersity index (PdI) of the ANC aqueous suspensions were measured using a dynamic light scattering (DLS) instrument (NanoZS, Malvern Panalytical, 
Malvern, UK). Each sample was diluted 1:50 in ultrapure water before measurements. Zeta potential was determined on the same sample with the same instrument. Measurements were made in triplicate at $25^{\circ} \mathrm{C}$.

\subsection{Bacterial and Fungal Organisms}

C. albicans ATCC $^{\circledR} 28367^{\text {TM }}$ and C. acnes ATCC ${ }^{\circledR} 6919$ were used for this study.

Yeasts were cultured on Sabouraud Glucose with Chloramphenicol agar plates aerobically at $37^{\circ} \mathrm{C}$ whereas C. acnes was cultured on Brain Heart Infusion (BHI) agar plates supplemented with $10 \%$ of defibrinated horse blood anaerobically at $37^{\circ} \mathrm{C}$. Before biofilm experiments, C. albicans and C. acnes were cultured overnight in $\mathrm{BHI}$ at $37^{\circ} \mathrm{C}$ in aerobic and anaerobic conditions, respectively. Following incubation, cultures were washed with PBS (centrifugation at $2000 \times g, 10 \mathrm{~min}$ ) and adjusted to $2 \times 10^{7}$ cells $/ \mathrm{mL}$ and $2 \times 10^{8}$ cells $/ \mathrm{mL}$ in fresh $\mathrm{BHI}$ for C. albicans and C. acnes respectively.

\subsection{Antibiofim Formation Assay}

Single-species C. albicans, single-species C. acnes and polymicrobial C. albicans-C. acnes biofilms were formed in 96-wells flat bottom nontreated polystyrene microplates. In the single-species condition, wells received $100 \mu \mathrm{L}$ of microbial suspensions. In polymicrobial condition, wells received $50 \mu \mathrm{L}$ of both microbial suspensions.

Antibiofilm formation activities of lipid extracts previously dissolved in DMSO were tested at two concentrations- 0.1 and $0.2 \mathrm{mg} / \mathrm{mL}$. Final DMSO concentrations did not exceed $2 \%$ of the overall volume in wells. For extracts included in nanocarriers (NCs), nanosystem tested concentrations were chosen to display extracts at 0.1 and $0.2 \mathrm{mg} / \mathrm{mL}$ in ultrapure water- "extract equivalent in nanocarrier" (mg/mL_EENC). Finally, empty nanocarriers were tested as controls and the studied concentrations corresponded to those present in nanocarriers loaded with extracts at 0.1 and $0.2 \mathrm{mg} / \mathrm{mL}(0.1$ _emptyNC and 0.2_emptyNC). A total of $100 \mu \mathrm{L}$ of extract or nanosystem solutions diluted in BHI were then added to the wells. Some wells without extract or nanosystem solution were reserved as a control and received $100 \mu \mathrm{l}$ of fresh $\mathrm{BHI}$ (BHI control). Microplates containing only C. albicans were incubated $24 \mathrm{~h}$ at $37^{\circ} \mathrm{C}$ in aerobic conditions while microplates containing C. acnes or both microorganisms were incubated in anaerobic conditions.

After incubation, cell concentrations were determined using a protocol adapted from the work of Kerstens et al., 2015 [51]. Planktonic cells were eliminated (2 rounds of washing with $200 \mu \mathrm{L}$ of PBS) and sessile cells were scraped off from the microplate bottom using sterile tips. An extensive rinsing of the microplate bottom was performed to detach remaining microorganisms. The obtained suspensions were sonicated for $10 \mathrm{~min}$ to break down aggregates (Elmasonic S 30, Elma Electronic, Wetzikon, Switzerland, $37 \mathrm{~Hz}$ ). This procedure has no effect on both C. albicans and C. acnes viability according to literature data [18,51].

In these microbial suspensions, cells concentrations were determined using flow cytometry (FCM). For dual-species conditions, FCM allowed us to distinguish the yeast population from that of bacteria according to their respective sizes and morphologies [22]. Measurements were performed on a CytoFLEX (Beckman Coulter, Indianapolis, IN USA) managed by CytExpert 2.0.0.153 software (Beckman Coulter, Indianapolis, IN, USA) and equipped with a blue laser $\left(\lambda_{\mathrm{ex}}=488 \mathrm{~nm}\right)$ and a $488 / 8$ bandpass filter. The flow rate used was $30 \mu \mathrm{L} \cdot \mathrm{min}^{-1}$.

\subsection{Anti-Preformed Biofilm Assay}

Single-species C. albicans, single-species C. acnes and polymicrobial C. albicans-C. acnes biofilms were formed in 96-well flat-bottom nontreated polystyrene microplates. In single-species condition, wells received $100 \mu \mathrm{L}$ of microbial suspensions. In polymicrobial condition, wells received $50 \mu \mathrm{L}$ of both microbial suspensions. Final volume was adjusted to $200 \mu \mathrm{L}$ using fresh BHI in all conditions. Microplates containing only $\mathrm{C}$. albicans were incubated $24 \mathrm{~h}$ at $37^{\circ} \mathrm{C}$ in aerobic conditions whereas microplates containing $C$. acnes or both microorganisms were incubated in anaerobic conditions. 
After incubation, supernatants were removed, and biofilms were carefully rinsed twice with $200 \mu \mathrm{L}$ of PBS. A total of $100 \mu \mathrm{L}$ of fresh BHI was added to all wells. Then, wells received $100 \mu \mathrm{L}$ of extract or nanosystem solutions diluted in BHI. Tested conditions were similar to those presented for antibiofilm formation assays. Some wells without extract or nanosystem solution were reserved as a control. A total of $100 \mu \mathrm{L}$ of fresh BHI was also used in control wells. Microplates containing only C. albicans were incubated $24 \mathrm{~h}$ at $37^{\circ} \mathrm{C}$ in aerobic conditions whereas microplates containing C. acnes or both microorganisms were incubated in anaerobic conditions.

After incubation, cell concentrations were determined as described previously for the antibiofilm formation assay.

\subsection{Statistical Analysis}

Experiments were performed at least in duplicate with four replicates for each condition. Mann-Whitney $U$ test was applied to determine statistical significance of the differences between the groups using GraphPad Prism ${ }^{\circledR}$ version 6.01 (GraphPad Software Inc, San Diego, CA USA). Differences were considered significant if $p<0.005$.

Author Contributions: Conceptualization, C.I., E.M., M.G. and L.B.-D.; Methodology, C.B., M.G., L.B.-D., E.M. and C.I.; Resources, B.C.L., M.G., L.B.-D., E.M. and C.I.; Investigation, V.L., C.B. and C.L.-L.; Formal analysis, C.B., E.M., L.B. and C.I.; Writing-Original Draft Preparation, C.B., L.B.-D., E.M. and C.I. and S.M.; Writing-Review \& Editing, C.B., B.C.-L., M.G., L.B.-D., E.M. and C.I.; Supervision, L.B.-D, E.M. and C.I.; Project administration, L.B.-D., E.M. and C.I. All authors have read and agree to the published version of the manuscript.

Funding: This work was supported by a grant (2018-2019) of the AAP Collaborative Research Action of the universities of Tours and Poitiers; This work was also supported by the 2015-2020 State-Region Planning Contracts (CPER), European Regional Development Fund (FEDER), and intramural funds from the Centre National de la Recherche Scientifique (CNRS) and the University of Poitiers. The authors thanks the ARD Cosmétosciences and the Centre Val de Loire Region for financial support (ARD 2017-00118114).

Acknowledgments: The authors also wish to thank Didier Debail and Garry Holding for revising the English text.

Conflicts of Interest: The authors have nothing to declare.

\section{References}

1. Bowler, P.G.; Duerden, B.I.; Armstrong, D.G. Wound microbiology and associated approaches to wound management. Clin. Microbiol. Rev. 2001, 14, 244-269. [CrossRef] [PubMed]

2. Kalan, L.; Grice, E.A. Fungi in the wound microbiome. Adv. Wound Care 2018, 7, 247-255. [CrossRef] [PubMed]

3. Johnson, T.R.; Gómez, B.I.; McIntyre, M.K.; Dubick, M.A.; Christy, R.J.; Nicholson, S.E.; Burmeister, D.M. The cutaneous microbiome and wounds: New molecular targets to promote wound healing. Int. J. Mol. Sci. 2018, 19. [CrossRef] [PubMed]

4. Vindenes, H.; Bjerknes, R. Microbial colonization of large wounds. Burns 1995, 21, 575-579. [CrossRef]

5. James, G.A.; Swogger, E.; Wolcott, R.; Pulcini, E.D.; Secor, P.; Sestrich, J.; Costerton, J.W.; Stewart, P.S. Biofilms in chronic wounds. Wound Repair Regen. 2008, 16, 37-44. [CrossRef]

6. Duerden, B.I. Virulence Factors in Anaerobes. Clin. Infect. Dis. 1994, 18, S253-S259. [CrossRef]

7. Mangram, A.J.; Horan, T.C.; Pearson, M.L.; Silver, L.C.; Jarvis, W.R. Guideline for Prevention of Surgical Site Infection, 1999. Centers for Disease Control and Prevention (CDC) Hospital Infection Control Practices Advisory Committee. Am. J. Infect. Control 1999, 27, 97-134. [CrossRef]

8. Noble, W.C. Skin microbiology: Coming of age. J. Med. Microbiol. 1984, 17, 1-12. [CrossRef]

9. Peleg, A.Y.; Hogan, D.A.; Mylonakis, E. Medically important bacterial-fungal interactions. Nat. Rev. Microbiol. 2010, 8, 340-349. [CrossRef]

10. Scholz, C.F.P.; Kilian, M. The natural history of cutaneous propionibacteria, and reclassification of selected species within the genus Propionibacterium to the proposed novel genera Acidipropionibacterium gen. Nov., Cutibacterium gen. nov. and Pseudopropionibacterium gen. nov. Int. J. Syst. Evol. Microbiol. 2016, 66, 4422-4432. [CrossRef]

11. Grice, E.A.; Segre, J.A. The skin microbiome. Nat. Rev. Microbiol. 2011, 9, 244-253. [CrossRef] [PubMed] 
12. Cogen, A.L.; Nizet, V.; Gallo, R.L. Skin microbiota: A source of disease or defence? Br. J. Dermatol. 2008, 158, 442-455. [CrossRef] [PubMed]

13. Kuehnast, T.; Cakar, F.; Weinhäupl, T.; Pilz, A.; Selak, S.; Schmidt, M.A.; Rüter, C.; Schild, S. Comparative analyses of biofilm formation among different Cutibacterium acnes isolates. Int. J. Med. Microbiol. 2018, 308, 1027-1035. [CrossRef] [PubMed]

14. Achermann, Y.; Goldstein, E.J.C.; Coenye, T.; Shirtliffa, M.E. Propionibacterium acnes: From Commensal to opportunistic biofilm-associated implant pathogen. Clin. Microbiol. Rev. 2014, 27, 419-440. [CrossRef]

15. Bernard, C.; Lemoine, V.; Hoogenkamp, M.A.; Girardot, M.; Krom, B.P.; Imbert, C. Candida albicans enhances initial biofilm growth of Cutibacterium acnes under aerobic conditions. Biofouling 2019, 35, 350-360. [CrossRef]

16. Tyner, H.; Patel, R. Propionibacterium acnes biofilm-A sanctuary for Staphylococcus aureus? Anaerobe 2016, 40, 63-67. [CrossRef]

17. Bojar, R.A.; Holland, K.T. Acne and Propionibacterium acnes. Clin. Dermatol. 2004, 22, 375-379. [CrossRef]

18. Bayston, R.; Ashraf, W.; Barker-Davies, R.; Tucker, E.; Clement, R.; Clayton, J.; Freeman, B.J.C.; Nuradeen, B. Biofilm formation by Propionibacterium acnes on biomaterials in vitro and in vivo: Impact on diagnosis and treatment. J. Biomed. Mater. Res. Part A 2007, 81A, 705-709. [CrossRef]

19. Coenye, T.; Peeters, E.; Nelis, H.J. Biofilm formation by Propionibacterium acnes is associated with increased resistance to antimicrobial agents and increased production of putative virulence factors. Res. Microbiol. 2007, 158, 386-392. [CrossRef]

20. Kuhn, D.M.; George, T.; Chandra, J.; Mukherjee, P.K.; Ghannoum, M.A. Antifungal susceptibility of Candida biofilms: Unique efficacy of amphotericin B lipid formulations and echinocandins. Antimicrob. Agents Chemother. 2002, 46, 1773-1780. [CrossRef]

21. Mukherjee, P.K.; Chandra, J. Candida biofilm resistance. Drug Resist. Updat. 2004, 7, 301-309. [CrossRef] [PubMed]

22. Bernard, C.; Renaudeau, N.; Mollichella, M.L.; Quellard, N.; Girardot, M.; Imbert, C. Cutibacterium acnes protects Candida albicans from the effect of micafungin in biofilms. Int. J. Antimicrob. Agents 2018, 52, 942-946. [CrossRef] [PubMed]

23. Montelongo-Jauregui, D.; Srinivasan, A.; Ramasubramanian, A.K.; Lopez-Ribot, J.L. An in vitro model for oral mixed biofilms of Candida albicans and Streptococcus gordonii in synthetic saliva. Front. Microbiol. 2016, 7, 1-13. [CrossRef] [PubMed]

24. Desbois, A.P.; Smith, V.J. Antibacterial free fatty acids: Activities, mechanisms of action and biotechnological potential. Appl. Microbiol. Biotechnol. 2010, 85, 1629-1642. [CrossRef]

25. Ruffell, S.E.; Müller, K.M.; McConkey, B.J. Comparative assessment of microalgal fatty acids as topical antibiotics. J. Appl. Phycol. 2016, 28, 1695-1704. [CrossRef]

26. D'Alessandro, E.B.; Antoniosi Filho, N.R. Concepts and studies on lipid and pigments of microalgae: A review. Renew. Sustain. Energy Rev. 2016, 58, 832-841. [CrossRef]

27. Hess, S.K.; Lepetit, B.; Kroth, P.G.; Mecking, S. Production of chemicals from microalgae lipids-Status and perspectives. Eur. J. Lipid Sci. Technol. 2018, 120, 1700152. [CrossRef]

28. Kłodzińska, S.N.; Wan, F.; Jumaa, H.; Sternberg, C.; Rades, T.; Nielsen, H.M. Utilizing nanoparticles for improving anti-biofilm effects of azithromycin: A head-to-head comparison of modified hyaluronic acid nanogels and coated poly (lactic-co-glycolic acid) nanoparticles. J. Colloid Interface Sci. 2019, 555, 595-606. [CrossRef]

29. Sánchez, M.C.; Toledano-Osorio, M.; Bueno, J.; Figuero, E.; Toledano, M.; Medina-Castillo, A.L.; Osorio, R.; Herrera, D.; Sanz, M. Antibacterial effects of polymeric PolymP-n Active nanoparticles. An in vitro biofilm study. Dent. Mater. 2019, 35, 156-168. [CrossRef]

30. Boutin, R.; Munnier, E.; Renaudeau, N.; Girardot, M.; Pinault, M.; Chevalier, S.; Chourpa, I.; Clément-Larosière, B.; Imbert, C.; Boudesocque-Delaye, L. Spirulina platensis sustainable lipid extracts in alginate-based nanocarriers: An algal approach against biofilms. Algal Res. 2019, 37, 160-168. [CrossRef]

31. Nguyen, H.T.P.; Munnier, E.; Souce, M.; Perse, X.; David, S.; Bonnier, F.; Vial, F.; Yvergnaux, F.; Perrier, T.; Cohen-Jonathan, S.; et al. Novel alginate-based nanocarriers as a strategy to include high concentrations of hydrophobic compounds in hydrogels for topical application. Nanotechnology 2015, 26, 255101. [CrossRef] [PubMed]

32. Fulaz, S.; Vitale, S.; Quinn, L.; Casey, E. Nanoparticle-Biofilm Interactions: The Role of the EPS Matrix. Trends Microbiol. 2019, 27, 915-926. [CrossRef] 
33. Lopes, L.Q.S.; Santos, C.G.; de Almeida Vaucher, R.; Raffin, R.P.; Santos, R.C.V. Nanocapsules with glycerol monolaurate: Effects on Candida albicans biofilms. Microb. Pathog. 2016, 97, 119-124. [CrossRef] [PubMed]

34. Lohse, M.B.; Gulati, M.; Valle Arevalo, A.; Fishburn, A.; Johnson, A.D.; Nobile, C.J. Assessment and optimizations of Candida albicans in vitro biofilm assays. Antimicrob. Agents Chemother. 2017, 61. [CrossRef] [PubMed]

35. Taff, H.T.; Nett, J.E.; Andes, D.R. Comparative analysis of Candida biofilm quantitation assays. Med. Mycol. 2012, 50, 214-218. [CrossRef]

36. Dhale, R.P.; Ghorpade, M.V.; Dharmadhikari, C.A. Comparison of various methods used to detect biofilm production of Candida species. J. Clin. Diagn. Res. 2014, 8, DC18-c20. [CrossRef]

37. Juin, C.; Perrin, F.; Puy, T.; Bernard, C.; Mollichella, M.L.; Girardot, M.; Costa, D.; Guillard, J.; Imbert, C. Anti-biofilm activity of a semi-synthetic molecule obtained from resveratrol against Candida albicans biofilm. Med. Mycol. 2019. [CrossRef]

38. Harriott, M.M.; Noverr, M.C. Candida albicans and Staphylococcus aureus form polymicrobial biofilms: Effects on antimicrobial resistance. Antimicrob. Agents Chemother. 2009, 53, 3914-3922. [CrossRef]

39. Morales, D.K.; Hogan, D.A. Candida albicans interactions with bacteria in the context of human health and disease. PLoS Pathog. 2010, 6, 1-4. [CrossRef]

40. Harriott, M.M.; Noverr, M.C. Ability of Candida albicans mutants to induce Staphylococcus aureus vancomycin resistance during polymicrobial biofilm formation. Antimicrob. Agents Chemother. 2010, 54, 3746-3755. [CrossRef]

41. Kim, D.; Liu, Y.; Benhamou, R.I.; Sanchez, H.; Simon-Soro, A.; Li, Y.; Hwang, G.; Fridman, M.; Andes, D.R.; Koo, H. Bacterial-derived exopolysaccharides enhance antifungal drug tolerance in a cross-kingdom oral biofilm. ISME J. 2018, 12, 1427-1442. [CrossRef] [PubMed]

42. Bernard, C.; Girardot, M.; Imbert, C. Candida albicans interaction with Gram-positive bacteria within interkingdom biofilms. J. Mycol. Med. 2019. [CrossRef]

43. Adam, B.; Baillie, G.S.; Douglas, L.J. Mixed species biofilms of Candida albicans and Staphylococcus epidermidis. J. Med. Microbiol. 2002, 51, 344-349. [CrossRef] [PubMed]

44. Cheong, Y.-K.; Arce, M.P.; Benito, A.; Chen, D.; Luengo Crisóstomo, N.; Kerai, L.V.; Rodríguez, G.; Valverde, J.L.; Vadalia, M.; Cerpa-Naranjo, A.; et al. Synergistic Antifungal Study of PEGylated Graphene Oxides and Copper Nanoparticles against Candida albicans. Nanomaterials 2020, 10. [CrossRef] [PubMed]

45. Punniyakotti, P.; Panneerselvam, P.; Perumal, D.; Aruliah, R.; Angaiah, S. Anti-bacterial and anti-biofilm properties of green synthesized copper nanoparticles from Cardiospermum halicacabum leaf extract. Bioprocess Biosyst. Eng. 2020, 1-9. [CrossRef]

46. Anjum, A.; Chung, P.Y.; Ng, S.F. PLGA/xylitol nanoparticles enhance antibiofilm activity: Via penetration into biofilm extracellular polymeric substances. RSC Adv. 2019, 9, 14198-14208. [CrossRef]

47. Tan, Y.; Ma, S.; Leonhard, M.; Moser, D.; Ludwig, R.; Schneider-Stickler, B. Co-immobilization of cellobiose dehydrogenase and deoxyribonuclease I on chitosan nanoparticles against fungal/bacterial polymicrobial biofilms targeting both biofilm matrix and microorganisms. Mater. Sci. Eng. C 2020, 108, 110499. [CrossRef]

48. Wang, T.; Bai, J.; Jiang, X.; Nienhaus, G.U. Cellular uptake of nanoparticles by membrane penetration: A study combining confocal microscopy with FTIR spectroelectrochemistry. ACS Nano 2012, 6, 1251-1259. [CrossRef]

49. Bautista-Hernández, L.A.; Gómez-Olivares, J.L.; Buentello-Volante, B.; Dominguez-Lopez, A.; Garfias, Y.; Acosta-García, M.C.; Calvillo-Medina, R.P.; Bautista-de Lucio, V.M. Negative interaction of Staphylococcus aureus on Fusarium falciforme growth ocular isolates in an in vitro mixed biofilm. Microb. Pathog. 2019, 135, 103644. [CrossRef]

50. Samburova, V.; Lemos, M.S.; Hiibel, S.; Kent Hoekman, S.; Cushman, J.C.; Zielinska, B. Analysis of triacylglycerols and free fatty acids in algae using ultra-performance liquid chromatography mass spectrometry. JAOCS J. Am. Oil Chem. Soc. 2013, 90, 53-64. [CrossRef]

51. Kerstens, M.; Boulet, G.; Van kerckhoven, M.; Clais, S.; Lanckacker, E.; Delputte, P.; Maes, L.; Cos, P. A flow cytometric approach to quantify biofilms. Folia Microbiol. 2015, 60, 335-342. [CrossRef] [PubMed]

(C) 2020 by the authors. Licensee MDPI, Basel, Switzerland. This article is an open access article distributed under the terms and conditions of the Creative Commons Attribution (CC BY) license (http://creativecommons.org/licenses/by/4.0/). 